\title{
Anomalous drift of spiral waves in heterogeneous excitable media
}

\author{
S. Sridhar ${ }^{1}$, Sitabhra Sinha ${ }^{1}$ and Alexander V. Panfilov ${ }^{2}$ \\ ${ }^{1}$ The Institute of Mathematical Sciences, CIT Campus, Taramani, Chennai 600113, India. and \\ ${ }^{2}$ Department of Theoretical Biology, Utrecht University, \\ Padualaan 8, Utrecht 3584 CH, The Netherlands
}

(Dated: October 2, 2018)

\begin{abstract}
We study the drift of spiral waves in a simple model of heterogeneous excitable medium, having gradients in local excitability or cellular coupling. For the first time, we report the anomalous drift of spiral waves towards regions having higher excitability, in contrast to all earlier observations in reaction-diffusion models of excitable media. Such anomalous drift can promote the onset of complex spatio-temporal patterns, e.g., those responsible for life-threatening arrhythmias in the heart.
\end{abstract}

PACS numbers: 87.19.Hh,05.45.-a,87.18.Hf,87.19.Lp

Spatial patterns of activity, in particular spiral waves, are observed in a broad class of physical, biological and chemical excitable systems [1]. One of the most important contexts in which spiral waves occur is that of electrical activity in the heart, where they can act as local sources of high-frequency excitations. This disrupts the rhythmic pumping action of the heart, leading to irregularities known as arrhythmias [2]. Understanding the dynamics of spiral waves may potentially result in improved methods for controlling such arrhythmias $3,3,4,[5,6,67,8]$. Spiral wave dynamics, primarily characterised by the motion of its core (i.e., the trajectory of the spiral wave tip, defined to be a phase singularity) can be either stationary rotation, or, evolving with time as in the case of meandering and drift 9]. Drift, which has a significant linear translational component, is a possible underlying mechanism for polymorphic ventricular tachycardia [4, 9]. This arrhythmia, which is characterised by an aperiodic electrocardiogram, can be a precursor of fully disordered activity that characterizes potentially fatal ventricular fibrillation [10]. Therefore, understanding the mechanisms leading to spiral wave drift is not only a problem of central interest for physics of excitable media, but also has potential clinical significance.

One of the most important causes of spiral drift is the heterogeneous nature of the excitable medium. This was first predicted in cellular automata models with step-like or discontinuous inhomogeneity [11], which was later confirmed by experiments [12, 13]. Subsequently, drift has been shown to be induced by a smooth gradient of excitability in both simple and biologically realistic ionic models of cardiac tissue [14, 15, 16]. Theoretical arguments indicate that the direction of the transverse component of the spiral drift (i.e., orthogonal to the gradient) depends on model parameters [17]. On the other hand, the longitudinal component is always directed towards the region with lower excitability (corresponding to longer periods of spiral rotation) [16]. This phenomenon has been seen in a variety of excitable media models of different complexity [14, 15, 16]. However, till date there is no satisfactory understanding of the reasons behind the spiral wave drift towards regions with longer rotation period. Although earlier kinematic stud- ies suggested the possibility of drift towards region with higher excitability [18], it has never actually been observed in a model of excitable tissue. The occurrence of drift in the direction of shorter period (corresponding to higher excitability) may have clinical significance, as it moves the spiral wave to a section where it rotates faster. This increases the likelihood of onset of additional wavebreaks away from the core, in the region where the medium is less excitable. Thus, it is a possible generation mechanism for "mother rotor" fibrillation [19, 20, 21], characterised by a stationary persistent source of highfrequency excitations giving rise to turbulent activity in the heart.

Electro-physiological heterogeneities in cardiac tissue may arise, in general, through spatial variation in the ionic currents of the excitable cells. There can also be gradients in the inter-cellular coupling as a result of the inhomogeneous distribution of the conductances of gap junctions connecting neighboring cells. In this paper, we use a simple model of cardiac tissue to investigate the role of both these types of heterogeneities in governing the direction of the spiral wave drift. We report the existence of a regime where the spiral wave core moves towards the region with higher excitability. This is a novel finding, never before observed in a model of excitable media and it may substantially increase the understanding of how heterogeneities affect spiral wave dynamics in the heart.

A generic model of excitable media that describes the dynamics of trans-membrane potential $V$ in cardiac tissue has the form

$$
\begin{gathered}
\partial V / \partial t=\nabla \gamma D \nabla V+\alpha I_{i o n}\left(V, g_{i}\right), \\
\partial g_{i} / \partial t=F\left(V, g_{i}\right) .
\end{gathered}
$$

Here, $I_{i o n}$ is the total ionic current traveling through the channels on the cellular membrane, $D$ accounts for the inter-cellular coupling and $g_{i}$ describes the dynamics of gating variables for the various ion channels. In this paper, we study the effects of heterogeneous distribution of the ionic currents and intercellular couplings. For this purpose, we introduce the parameters $\alpha$ and $\gamma$, which represent the spatial variation in ionic currents and conduction properties (respectively) for an inhomogeneous 
medium. Parameter $\alpha$ directly scales the value of the ionic current in Eq. 1, while $\gamma$ scales the diffusion coefficient as $D=D_{0}+\gamma(x)\left(D_{0}=1\right.$ for the rest of the paper). In this study, we have used the Barkley model [22], where the several gating variables are aggregated into a single variable $g$ that controls the slow recovery dynamics of the medium with $F(V, g)=V-g$. The nonlinear dependence of the ionic current on the fast variable $V$ is represented by the cubic function $I_{i o n}=[V(1-V)(V-((g+b) / a)] / \epsilon$, where $a$ and $b$ are parameters governing the local kinetics and $\epsilon$ is the relative time scale between the local dynamics of $V$ and $g$. The spatial heterogeneity of local excitability and cellular coupling are assumed to have linear functional form, viz., $\alpha(x)=\alpha_{0}+\Delta \alpha x$ and $\gamma(x)=\gamma_{0}+\Delta \gamma x$. The variable $x$ represents the spatial position along the principal direction of the inhomogeneity gradient, the origin being considered to be the initial position of the spiral wave tip. At this point, $\alpha=\alpha_{0}$, $\gamma=\gamma_{0}$, and $\Delta \alpha, \Delta \gamma$ measure their rate of change along the gradient. For all the figures in this paper, we have used $\alpha_{0}=1.15$ and $\gamma_{0}=1.3$.

The two dimensional system is discretized on a square spatial grid of size $L \times L(L=200$ for the figures shown here). The values of space step $\Delta x$ and time step $\Delta t$ used are 0.5 and 0.005 respectively. A sample of simulations have been repeated for $\Delta x=0.25$ to verify numerical accuracy. The model equations are solved using forward Euler scheme with a standard 5-point stencil for the Laplacian. No-flux boundary conditions are implemented at the edges of the simulation domain. The initial condition for all simulations is a stable non-meandering spiral, the spiral tip being at the centre of the simulation domain.

Increasing either the ionic current (via $\alpha$ ) or intercellular coupling (via $\gamma$ ) results in increasing the excitability of the medium. Thus, to investigate the role of heterogeneity in spiral drift, we have considered spatial gradients in $\alpha$ or $\gamma$ individually (keeping the other parameter constant). After extensive numerical simulations that scan over the $(a, b)$ parameter space of the Barkley model, we have found that it is indeed possible to observe anomalous drift of the spiral, i.e., drift towards regions with higher excitability (shorter periods). An example of such anomalous drift is shown in Fig. 1 $(A, C)$. For comparison, in Fig. 1](B,D) we show the normal drift of the spiral towards regions of lower excitability. This is seen for a set of $(a, b)$ values which is farther from the boundary with the sub-excitable region of the Barkley model 23] than the $(a, b)$ parameter set for which anomalous drift is observed in Fig. 1] (A,C).

To analyse the genesis of anomalous drift, we first look at how the parameters $\gamma$ and $\alpha$ affect the spiral wave in an homogeneous medium. As $\gamma$ is only a scaling factor for the diffusion coefficient, the period of the spiral wave does not depend on it. Further, scaling arguments suggest that the spiral wavelength increases as a square root of $\gamma$. Thus, for normal drift in the presence of a gradient in $\gamma$, the spiral moves towards the shorter wave-

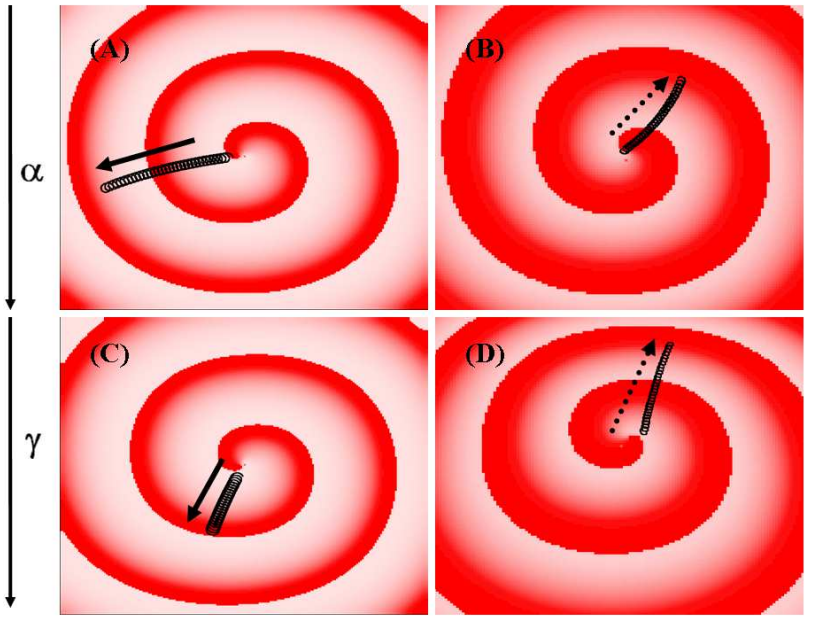

FIG. 1: Anomalous and normal drift of spiral wave. Pseudocolor images of spiral wave at the instant when the gradient in local excitability $\alpha$ (top row) and cellular coupling (bottom row) is applied. (A, C) Anomalous drift in the direction of higher excitability, i.e., towards increasing values of $\alpha$ (A) or $\gamma(\mathrm{C})$, the direction being shown by solid arrows. (B, D) Normal drift in $\alpha$ (B) or $\gamma(\mathrm{D})$ gradient. Parameter values are $a=0.82, b=0.13$ (for $\mathrm{A}, \mathrm{C}$ ) and $a=1.02, b=0.15$ (for B, D). The gradients applied are (A, B) $\Delta \alpha=0.0025$, $\Delta \gamma=0$, and (C, D) $\Delta \alpha=0, \Delta \gamma=0.020$. In all cases, the gradient is along the vertical direction, with $\alpha$ or $\gamma$ increasing from top to bottom. In $(\mathrm{B}, \mathrm{D})$ the region around the core is magnified to make the wavelength of the spiral comparable to that in $(\mathrm{A}, \mathrm{C})$.

length region, while for anomalous drift, it is directed towards longer wavelengths. Fig. 2 shows the variation of the spiral period and wavelength as a function of the parameter $\alpha$, both of which decrease as $\alpha$ increases [24]. From these results we can infer that, for normal drift in the presence of $\alpha$ gradient, the period and wavelength of the spiral increase as the core moves towards lower $\alpha$ regions. In contrast, we see a decrease in the period and wavelength in the case of anomalous drift towards regions having higher values of $\alpha$.

Next, we study the effect of the magnitude of spatial gradient in $\alpha$ or $\gamma$ on the velocity of spiral drift. Fig. 3 shows the longitudinal component of the drift velocity, $V_{L}$, i.e., along the gradient, as a function of the spatial variation in $\alpha$ or $\gamma$. Note that, positive $V_{L}$ corresponds to anomalous, while, negative $V_{L}$ corresponds to normal drift of the spiral wave. Fig. 3 shows that, for normal drift, increasing either of the gradients results in a monotonic increase of $V_{L}$. However, in the case of anomalous drift as a result of $\alpha$ gradient, we see a non-monotonic behavior in $V_{L}$, which first increases but then decreases and becomes negative (Fig. 3, a). Thus, the anomalous drift of the spiral towards higher excitability in $\alpha$ gradient is seen only for small $\Delta \alpha$. For higher $\Delta \alpha$, there is a reversal of direction and the spiral exhibits normal drift. On the other hand, Fig. 3 (b) shows that for a gradient in $\gamma$, the anomalous drift is observed for the entire range of $\Delta \gamma$ that is investigated. 

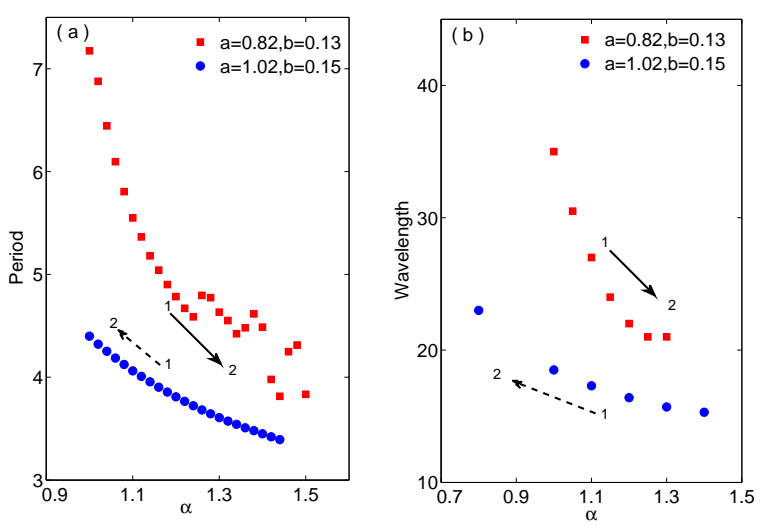

FIG. 2: The variation of spiral period and wavelength as a function of the parameter $\alpha$. The symbols " 1 " and " 2 " correspond to the values of $\alpha$ in the region around the initial and final positions (respectively) of the spiral waves in Fig. 1] with the same sets of Barkley model parameters being used. The solid and broken arrows represent the directions of anomalous and normal drift, respectively, in presence of a gradient in $\alpha$.
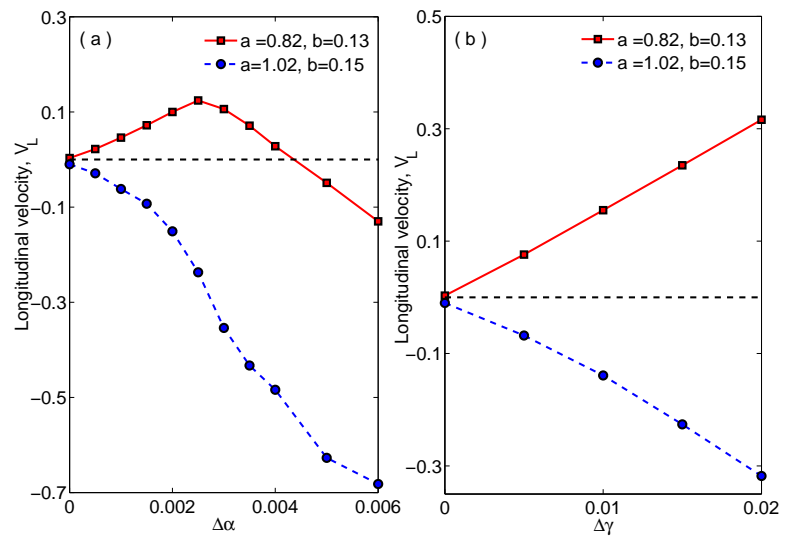

FIG. 3: Drift velocity depends on the gradient in parameters $\alpha$ and $\gamma$. (a) Non-monotonic variation (solid curve) of the longitudinal component of spiral wave drift velocity $V_{L}$ as a function of the gradient in local excitability, $\Delta \alpha$, for a model system with parameters $a=0.82, b=0.13$. Positive values of $V_{L}$ indicate anomalous drift. For a different set of parameters $(a=1.02, b=0.15)$, normal drift is seen for the entire range of gradients used (broken curve). (b) Variation of $V_{L}$ with the gradient in cellular coupling, $\Delta \gamma$. Solid and broken curves represent the anomalous and normal drift seen for the two parameter sets mentioned earlier (respectively), and are observed throughout the range of gradients used.

We have also studied the effect of the local kinetics on anomalous drift by varying the Barkley model parameter $a$ (Fig. 4, a). Increasing $a$ (keeping $b$ fixed) decreases the activation threshold of the medium, and thus makes the system more excitable. We observe that for both $\alpha$ and $\gamma$ gradients, the variation of $V_{L}$ as a function of $a$ is non-monotonic. For the cellular coupling $(\gamma)$ gradient, the presence of anomalous regime clearly correlates with excitability. The drift is anomalous at lower excitabil-
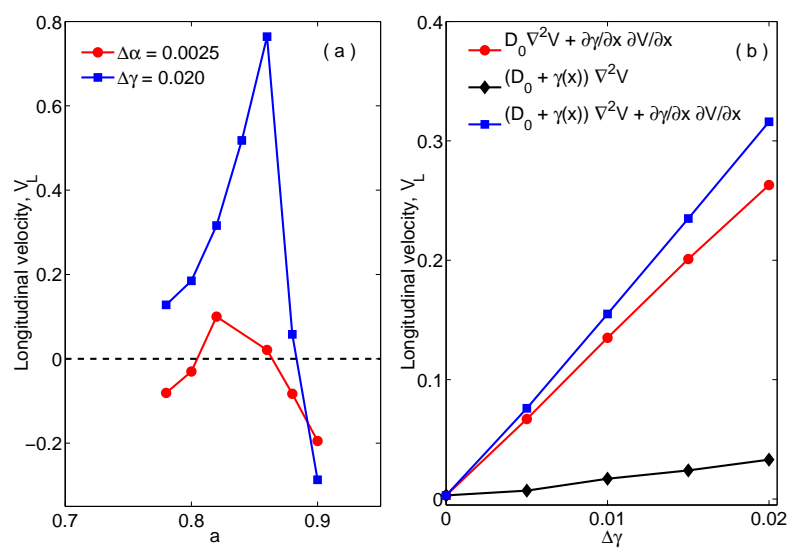

FIG. 4: (a) Non-monotonic variation of the longitudinal component of drift velocity, $V_{L}$, as a function of the model parameter $a$. The two curves correspond to media having a constant gradient in excitability (circles: $\Delta \alpha=0.0025, \Delta \gamma=0$ ) and cellular coupling (squares: $\Delta \alpha=0, \Delta \gamma=0.020$ ). For both, the parameter $b=0.13$. (b) The contribution to $V_{L}$ from the different components in the diffusion term as a function of the gradient in cellular coupling, $\nabla \gamma$. The circles and diamonds correspond to the linear and second-order contributions, and, squares correspond to the complete Laplacian term, respectively. All data points shown are for $a=0.82, b=0.13$.

ity, but becomes normal at higher excitability. However, for the gradient in $\alpha$, the anomalous drift occurs only over an intermediate range of $a$. For lower and higher excitabilities, the drift becomes normal.

The mechanism of anomalous drift in $\alpha$ gradient remains unclear. However, we can understand the anomalous drift for the cellular coupling $(\gamma)$ gradient, by relating it to other drift phenomena in excitable media. Note that, the Laplacian term in Eq. (11) can be expanded as,

$$
\nabla \gamma(x) D \nabla V=\left(D_{0}+\gamma(x)\right) \nabla^{2} V+\partial \gamma / \partial x \partial V / \partial x .
$$

Therefore, the heterogeneous cellular coupling $\gamma(x)$ contributes to both the gradient $(\partial \gamma / \partial x \partial V / \partial x)$, as well as, second order $\left(\gamma(x) \nabla^{2} V\right)$ terms. The relative contributions of these terms to the longitudinal component of drift velocity is shown in Fig. 目 (b). We observe that the principal effect on $V_{L}$ is due to the $\partial \gamma / \partial x \partial V / \partial x$ term, while $\gamma(x) \nabla^{2} V$ accounts only for about $10 \%$ of the observed drift. This allows us to propose the following explanation for anomalous drift in the presence of a gradient in $\gamma$. If we do not consider the $\gamma(x) \nabla^{2} V$ term in the Laplacian, the spatial operators in Eq. (3) are seen to be identical to those in equations describing drift of a spiral wave in the presence of an electric field [25]. This latter, in turn, is similar to the Laplacian describing the drift of radially symmetric filaments of a scroll ring in three-dimensional excitable media [26, 27]. As shown in Refs. [28, 29], the drifts observed in these two kinds of systems are induced by the same instabilities. We see from Fig. 目 (b) that the gradient $\partial \gamma / \partial x \partial V / \partial x$, which determines the drift in an electric field and that of scroll wave filaments, also determines the drift as a result of 
$\gamma$ gradient. Therefore, we infer that the anomalous drift direction (towards higher excitability) observed by us is also a result of the same long wavelength instabilities determining the drift of scroll wave filaments. This suggests that the occurrence of scroll expansion in 3-D implies the existence of anomalous drift in $\gamma$ gradient in 2-D. Conversely, observation of anomalous drift might suggest parameter regions where scroll wave expansion is possible.

In this paper, we have explicitly demonstrated the possibility of spiral waves to drift towards region of higher excitability in a simple model of heterogeneous excitable medium. Most of the detailed ionic models for cardiac tissue have the same form as Eq. (1) and, therefore, our analysis can be easily extended to biologically realistic models, such as LR1 or TNNP 30, 31. It might be possible to infer the parameter range in realistic models where anomalous drift may occur by using the relation between the cellular coupling gradient induced drift and scroll ring expansion. Note that, the latter phenomenon has recently been seen in the LR1 model [27].

Spiral waves are not only relevant for cardiac tissue, but are also observed in many different excitable media. Thus, it may be possible to relate our observation with results of kinematic studies 18] and models of cyclic catalysis in replicating entities [32], which predict drift towards region with shorter periods. From a clinical perspec- tive, anomalous drift is important as it clearly promotes arrhythmia and may result in fibrillation by promoting wave-breaks away from the spiral core. Spiral drift in the presence of a cellular coupling gradient maybe a key factor giving rise to abnormal wave activity in regions of the heart where conductivity changes abruptly, e.g., at Purkinje-muscle cell junctions or in an infarct border zone [33]. It can also be studied experimentally and numerically in many model systems, such as, heterogeneous mono-layers of neonatal rat cardiomyocytes [34].

To conclude, we have observed that spiral waves in heterogeneous excitable media can drift towards regions having higher excitability. Such anomalous drift occurs either in media having intermediate to low excitability when the heterogeneity is a gradient in ionic current, or, in media with low excitability for a gradient in the cellular coupling. Further, it appears to be related to regimes where expansion of 3-dimensional scroll wave filaments is observed. Anomalous drift of spiral waves may increase the likelihood of the onset of complex spatio-temporal patterns in excitable medium, e.g., turbulent electrical activity in the heart.

This work was supported in part by Utrecht University 2008 Short-Stay Fellowship, IMSc Complex Systems Project (XI Plan) and IFCPAR Project 3404-4.
[1] J. Keener and J. Sneyd, Mathematical Physiology (Springer, New York) (1998).

[2] J. M Davidenko, et al, Nature 355, 349 (1991).

[3] S. Sinha, A. Pande and R. Pandit, Phys. Rev. Lett. 86, 3678 (2001); S. Sridhar and S. Sinha, Europhys. Lett. 81, 50002 (2008).

[4] R. A. Gray et al, Circulation 91, 2454 (1995).

[5] D. Barkley, Phys. Rev. Lett. 68, 2090 (1992).

[6] T. K. Shajahan, S. Sinha and R. Pandit, Phys. Rev. E 75, 011929 (2007).

[7] A. V. Panfilov and A. V. Holden, J. Theor. Biol. 161, 271 (1993).

[8] A. V. Panfilov and J. P. Keener, J. Theor. Biol. 163, 439 (1993).

[9] A. Garfinkel and Z. Qu, in Cardiac Eletrophysiology: From Cell to Bedside, edited by D. P. Zipes and J. Jalife (Saunders, Philadelphia, 2004), p. 327.

[10] S. M. Cobbe and A. C. Rankin, in Oxford Textbook of Medicine, edited by D. A. Warrell, T. M. Cox and J. D. Firth and E. J. Benz (Oxford University Press, USA, 2005), p. 975.

[11] V. I. Krinsky, Problemy Kibernetiki 2, 59 (1968).

[12] V. G. Fast and A. M. Pertsov, Biophysics 35, 489 (1990).

[13] M. Markus, Zh. Nagy-Ungavarai and B. Hess, Science 257, 225 (1992).

[14] A. V. Panfilov and B. N. Vasiev, Physica D 49, 107 (1991).

[15] A. N. Rudenko and A. V. Panfilov, Stud. Biophysics 1, 183 (1983).

[16] K. H. W. J. ten Tusscher and A. V. Panfilov, Am. J. Physiol. Heart. Circ. Physiol 284, H542 (2003).
[17] G. R. Ivanitsky, V. I. Krinsky, A. V. Panfilov and M. A. Tsiganov, Biofizika 34, 297 (1989).

[18] A. S. Mikhailov, V. A. Davydov and V. S. Zykov, Physica D 70, 1 (1994).

[19] R. A. Gray, A. M. Pertsov and J. Jalife, Circulation 94, 2649 (1996).

[20] J. Jalife et al, Cardiovasc. Res. 54, 204 (2002).

[21] R. H. Keldermann et al, Am. J. Physiol. Heart Circ. Physiol. 296, H370 (2009).

[22] D. Barkley et al, Phys. Rev. A 42, 2489 (1990).

[23] A. Pumir et al, arXiv:0902.3891

[24] For $a, b$ parameters where anomalous drift is observed, the period and wavelength of spiral wave exhibits a more rapid divergence with decreasing $\alpha$ compared to the parameter regime showing normal drift.

[25] V. I. Krinsky, E. Hamm and V.Voignier, Phys. Rev. Lett 76, 3854 (1996).

[26] A. V. Panfilov and A. N. Rudenko, Physica 28D, 215 (1987).

[27] S. A. Alonso and A. V. Panfilov, Phys. Rev. Lett 100, 218101 (2008).

[28] H. Henry and V. Hakim, Phys. Rev. E 65, 046235 (2002).

[29] H. Henry, Phys. Rev. E 70, 026204 (2004).

[30] C. Luo and Y. Rudy, Circ. Res. 68, 1501 (1991).

[31] K. H. W. J Ten Tusscher, D. Noble, P. J. Noble and A. V. Panfilov, Am. J. Physiol. Heart. Circ. Physiol. 286, H1573 (2004).

[32] M. C. Boerlijst and P. Hogeweg, Physica D 88, 29 (1995).

[33] A. Pumir et al, Biophys. J. 89, 2332 (2005).

[34] V. N. Biktashev et al, Biophys. J. 94, 37262009. 